Original Article

\title{
AUTHENTICATION OF TURMERIC USING PROTON-NUCLEAR MAGNETIC RESONANCE SPECTROSCOPY AND MULTIVARIATE ANALYSIS
}

\author{
ANJAR WINDARSIH ${ }^{1}$, ABDUL ROHMAN $^{*}{ }^{*}$, RESPATI TRI SWASONO ${ }^{2}$
}

1Department of Pharmaceutical Chemistry, Faculty of Pharmacy, Universitas Gadjah Mada, Yogyakarta, ${ }^{2}$ Department of Chemistry, Faculty of Mathematics and Natural Sciences, Universitas Gadjah Mada, Yogyakarta

Email: abdulkimfar@gmail.com

Received: 08 Aug 2018, Revised and Accepted: 12 Sep 2018

\section{ABSTRACT}

Objective: The objective of this study was to apply ${ }^{1} \mathrm{H}-\mathrm{NMR}$ spectroscopy-based metabolite fingerprinting in combination with multivariate analysis for authentication of turmeric (Curcuma longa) from C. heyneana and C. manga.

Methods: Partial least square-discriminant analysis (PLS-DA) and orthogonal projections to latent structures-discriminant analysis (OPLS-DA) were used for differentiation of authentic and adulterated $C$. longa with $C$. manga and $C$. heyneana. The variables used were peaks with certain chemical shifts at optimized $1 \mathrm{H}-\mathrm{NMR}$ spectra of authentic and adulterated $C$. longa.

Results: All of the authentic $C$. longa samples were clearly separated from the adulterated ones. The multivariate calibration of partial least square (PLS) was successfully applied to predict of adulterants in C. longa. The lower RMSEC (root mean square error of calibration) values, $0.94 \%$ for adulterated $C$. longa with $C$. heyneana and $1.37 \%$ for adulterated $C$. longa with $C$. manga, and the lower RMSEP (root mean square error of prediction) values, $0.83 \%$ for adulterated $C$. longa with $C$. heyneana and $1.34 \%$ for adulterated $C$. longa with $C$. manga indicated the good of accuracy and precision of the calibration models.

Conclusion: The combination of ${ }^{1} \mathrm{H}-\mathrm{NMR}$ spectroscopy and chemometrics of multivariate analysis PLS-DA, OPLS-DA, and PLS proves an adequate technique for authentication of turmeric.

Keywords: ${ }^{1} \mathrm{H}-\mathrm{NMR}$ spectroscopy, Turmeric, Authentication, PLS-DA, OPLS-DA, PLS

(c) 2018 The Authors. Published by Innovare Academic Sciences Pvt Ltd. This is an open-access article under the CC BY license (http://creativecommons.org/licenses/by/4.0/) DOI: http://dx.doi.org/10.22159/ijap.2018v10i6.29014

\section{INTRODUCTION}

Curcuma longa is one of the herbaceous perennial used for a long time in the field of foodstuff, cosmetics, and traditional medicine [1], with several pharmacological activities of antioxidant [2], antiinflammatory [3], antibacterial, hepatoprotective, cardioprotective $[4,5]$, maintain gastrointestinal tract, and chemoresistance activities [6] due to curcuminoids compound (curcumin, demethoxycurcumin, and bisdemethoxycurcumin). Adulteration of medicinal plant is a common practice in the world to gain more economical benefit [7]. Among other Curcuma species, Curcuma heyneana and Curcuma manga are very potential to be used as adulterants in C. longa because they have strong yellow color, lower price, and wide availability [8].

Several methods have been developed for authentication of $C$. longa including high-performance liquid chromatography $[9,10]$, thin layer chromatography [11], high-performance thin layer chromatography [12], ultra-performance liquid chromatography [13], and capillary electrophoresis [14] which are aimed to determine the curcuminoids content. However, these methods are destructive, are not simple in sample preparation (too laborious), are time-consuming, and require more chemicals. The nondestructive and reliable methods including ultraviolet-visible spectroscopy, Fourier Transform Raman spectroscopy, near infrared, attenuated total reflectance (mid-infrared) spectroscopy [15-17], and proton nuclear magnetic resonance ( $\left.{ }^{1} \mathrm{H}-\mathrm{NMR}\right)$ spectroscopy have been applied for authentication in foods and medicinal plants [18]. The latter, ${ }^{1} \mathrm{H}-\mathrm{NMR}$ spectroscopy offered great advantages in metabolomics study for authentication of medicinal plants $[19,20]$ especially in combination with chemometrics of partial least square-discriminant analysis (PLS-DA) and orthogonal projections to latent structures-discriminant analysis (OPLS-DA). In this study, a combination of ${ }^{1} \mathrm{H}-\mathrm{NMR}$ spectroscopy and multivariate analysis of pattern recognition and regression was carried out for the authentication of $C$. longa adulterated with C. heyneana and $C$. manga.

\section{MATERIALS AND METHODS}

\section{Sample collection and preparation}

Rhizomes sample of Curcuma longa, Curcuma heyneana, and Curcuma manga were collected from Yogyakarta and Central Java, Indonesia. The authentication (plant determination) of these rhizomes was performed in Department of Pharmaceutical Biology, Faculty of Pharmacy, Universitas Gadjah Mada, Yogyakarta, Indonesia. Rhizomes were cleaned and chopped into small pieces. The chopped rhizomes were air-dried for 5-6 d. The dried rhizomes were ground into powder. The adulterated powder of $C$. longa with $C$. heyneana and $C$. manga were prepared by mixing in binary mixtures using the adulterant concentration of $5,10,25$, $40,50,60$, and $75 \% \mathrm{wt} / \mathrm{wt}$.

\section{Sample extraction for ${ }^{1} \mathrm{H}-\mathrm{NMR}$ analysis}

The extraction method was adapted from Kim et al. [19] with slight modification. Approximately of $25 \mathrm{mg}$ of powder was weighed and put into $2 \mathrm{ml}$ centrifuge tube. Subsequently added with $0.5 \mathrm{ml}$ of methanol-D4 $\left(\mathrm{CD}_{3} \mathrm{OD}\right)$ and $0.5 \mathrm{ml}$ of $\mathrm{KH}_{2} \mathrm{PO} 4$ (phosphate) buffer 90 mmol pH 6.0 in $\mathrm{D}_{2} \mathrm{O}$ containing TSP (trimethylsilyl propionic acid-D4 sodium salt) $0.01 \%$. The mixtures were vortexed for $1 \mathrm{~min}$, ultrasonicated for $20 \mathrm{~min}$, and centrifuged at $13500 \mathrm{rpm}$ for $10 \mathrm{~min}$. Approximately of $800 \mathrm{ml}$ of supernatant was taken and transferred into NMR tube for NMR measurement.

\section{${ }^{1}$ H-NMR spectra measurement}

The ${ }^{1} \mathrm{H}-\mathrm{NMR}$ spectra were acquired on a $500 \mathrm{MHz}$ Jeol ECZ-R spectrometer. Each ${ }^{1} \mathrm{H}-\mathrm{NMR}$ spectrum was acquired using the field strength of $11.74736 \mathrm{~T}$ (500 MHz), and X_Offset was $5.0 \mathrm{ppm}$. The relaxation delay was $5 \mathrm{~s}$, and the total scans for each spectrum were 128 scans. 3-Trimethylsilyl propionic acid-D4 sodium salt (TSP) was used as an internal standard for the calibration of chemical shift in NMR signals.

\section{Data preprocessing and multivariate analysis}

Preprocessing of data namely phasing, baseline correction, normalization, binning, and scaling was performed using 
MestreNova 12.0 .0 software. The spectra were manually phase corrected, and the chemical shift of internal standard of TSP was set at $0.00 \mathrm{ppm}$. Baseline correction was carried out using a polynomial fit mode, and then the spectra were normalized using total area. Binning for every bin width of $0.04 \mathrm{ppm}$ was performed for each spectrum to reduce data dimensionality. For pattern recognition analysis, binning was carried out in the region of 0.00 $10.00 \mathrm{ppm}$ excluding the regions of residual water and solvent (methanol) while for partial least square analysis, binning was performed in the aromatic region (6.00-8.00 ppm). All data were Pareto scaled for multivariate analysis. Chemometrics of multivariate analysis was carried out using SIMCA 14 and Minitab 17 software. The variables used were the chemical shifts binned for every $0.04 \mathrm{ppm}$ using average sum mode. Chemometrics supervised pattern recognition of PLS-DA was used to differentiate authentic and adulterated $C$. longa with $C$. heyneana and $C$. manga while OPLS-DA was employed to maximize the differentiation between samples. The number of variables for PLS-DA and OPLSDA analysis was 238 variables. Chemometrics regression of PLS using 51 variables was applied for quantification of adulterant $(C$. heyneana and C. manga) in binary mixtures with C. longa. The PLSDA and OPLS-DA models were validated using the permutation test while the PLS model was validated using RMSEC, RMSEP, and leave one out cross-validation technique.

\section{RESULTS AND DISCUSSION}

Spectra analysis of Curcuma longa, Curcuma heyneana, and Curcuma manga

${ }^{1} \mathrm{H}$-NMR spectroscopy is capable of measuring spectra of $C$. longa, $C$. heyneana, and C. manga. Representative spectra in fig. 1 showed the spectra of authentic $C$. longa, $C$. heyneana, $C$. manga, and adulterated $C$. longa with $25 \%$ of adulterants concentration. The spectra of authentic $C$. longa, C. heyneana, and C. manga showed clear differences, and they can be differentiated visually. However, the spectra of adulterated $C$. longa with $25 \%$ of $C$. heyneana and $25 \%$ of $C$. manga showed a similar pattern with the spectra of authentic $C$. longa. It is very difficult to differentiate them and to state whether the sample is authentic or adulterated just by inspecting the spectra visually. Therefore, an adequate statistical method such as chemometrics is important to overcome this problem.

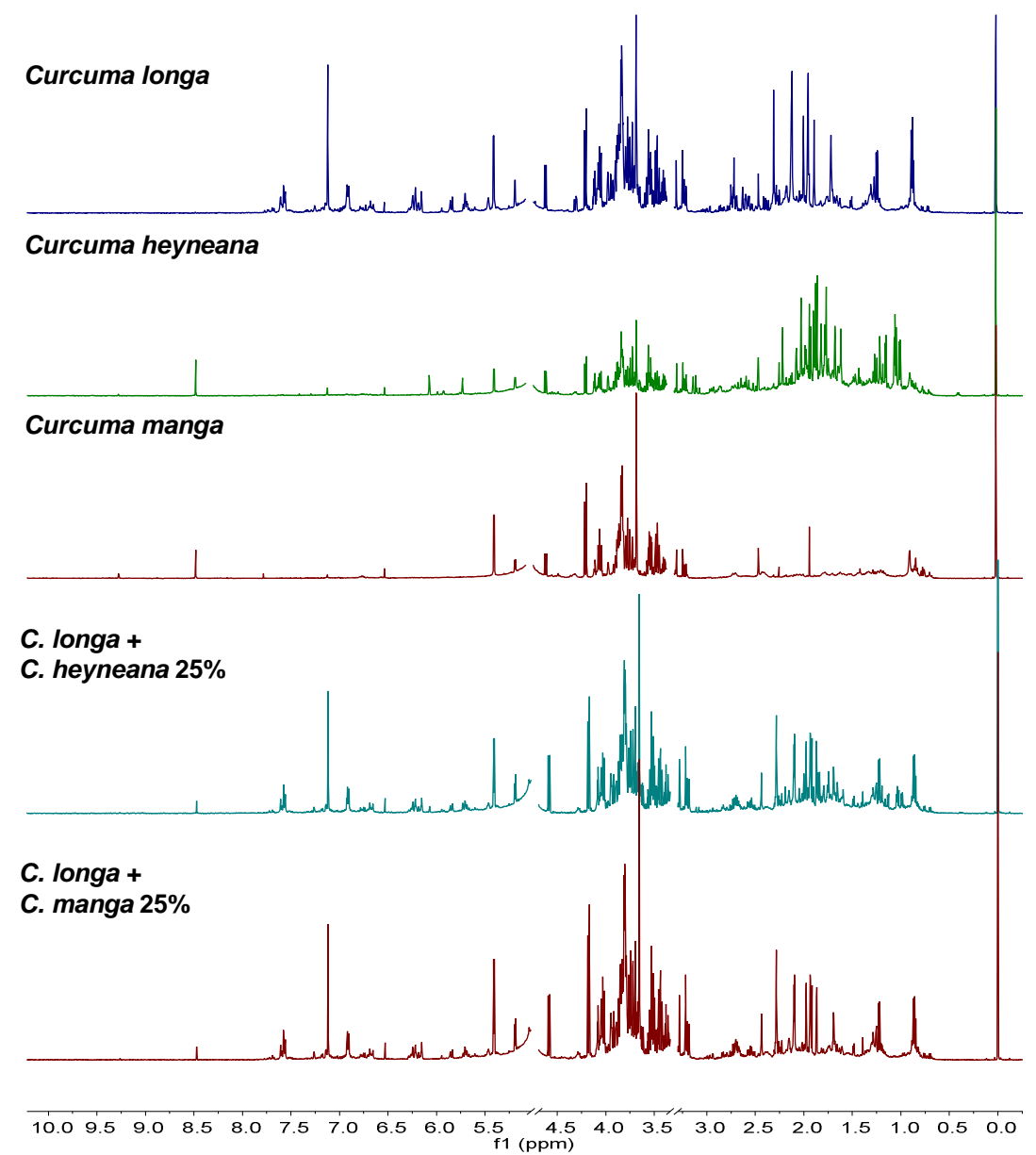

Fig. 1: ${ }^{1} \mathrm{H}$-NMR spectra of Curcuma longa, Curcuma heyneana, Curcuma manga, and adulterated Curcuma longa with $25 \%$ of adulterants concentration

Generally, the spectra can be divided into three regions, namely amino acid region (0.00-3.00 ppm), sugar region (3.00-5.50 ppm), and aromatic region $(6.00-8.00 \mathrm{ppm})[18,21]$. C. longa and $C$. heyneana have more intense signals in the amino acid region compared to $C$. manga while in the regions of aromatic, only C. longa showed intense signals. This corresponds to the high content of curcuminoids in $C$. longa especially curcumin than in $C$. heyneana and C. manga. Extracted using methanol-D4 and phosphate buffer in $\mathrm{D}_{2} \mathrm{O}$, curcumin appeared in the chemical shift of $7.28 \mathrm{ppm}$ (singlet), $3.90 \mathrm{ppm}$ (singlet), $6.80 \mathrm{ppm}$ and $7.22 \mathrm{ppm}$ (doublet), while demethoxycurcumin signals appeared in the chemical shift of 5.89 ppm (singlet), $3.94 \mathrm{ppm}$ (singlet), and $6.92 \mathrm{ppm}$ (doublet) [22, 23].

Adulteration affected curcuminoids content especially curcumin in C. longa due to the lower curcumin content in $C$. heyneana and $C$. manga. In the powder form, $C$. longa contains 5-6.5\% of curcumin, while curcumin content in C. heyneana is around $0.67-1.2 \%$ and in $C$. manga is not more than $0.05 \%$ [24]. Representative spectra in the aromatic region of authentic $C$. longa and adulterated $C$. long $a$ with $C$. heyneana in various adulterant concentrations were shown in fig. 2 . 
Deep investigation of stacked spectra of authentic and adulterated $C$. longa with various concentrations of $C$. heyneana in this region showed the decreasing of signal intensities in some areas. These correspond to the decreasing of curcuminoids content in adulterated samples. In addition, the spectra of authentic and adulterated $C$. longa with various concentrations of $C$. manga in the aromatic region also showed the decreasing of signal intensities in some areas as shown in fig. 3. The decreasing of signal intensities in this aromatic region (6.00-8.00 $\mathrm{ppm}$ ) as the increasing of adulterants concentration might be caused by the decreasing of curcuminoids content in the mixture because of the lower concentration of curcuminoids in $C$. heyneana and $C$. manga. Therefore, this aromatic region can be used to build a chemometrics model for quantification of adulterants in assessing the authenticity of $C$. longa.

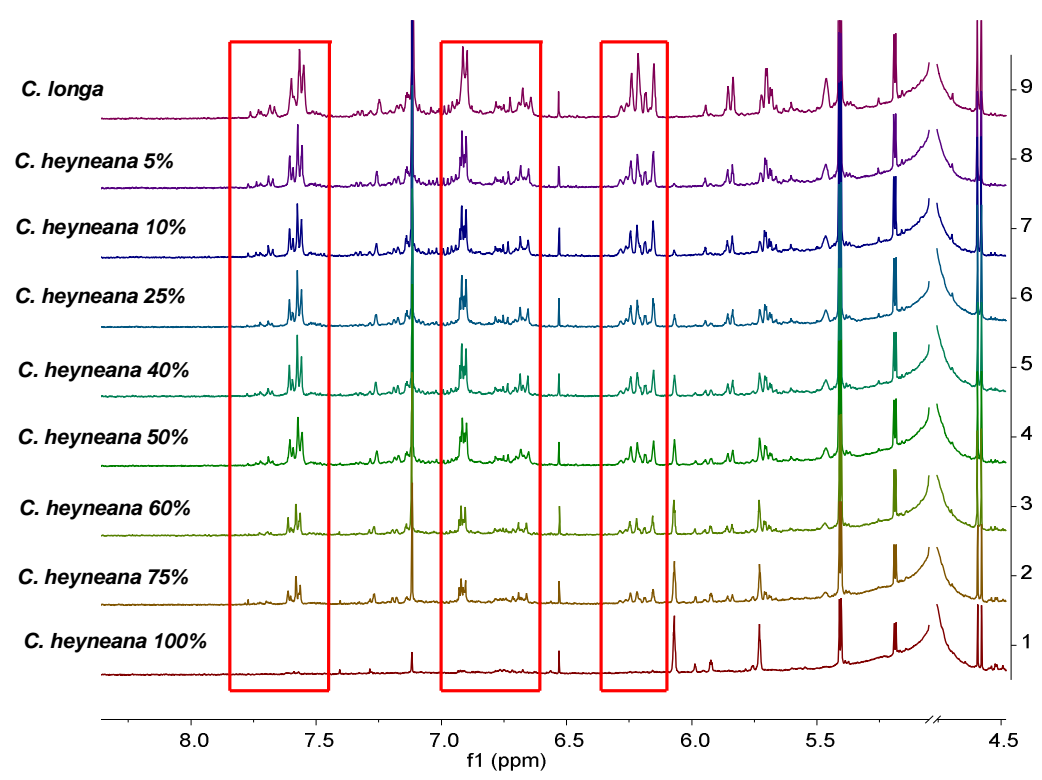

Fig. 2: ${ }^{1} \mathrm{H}-\mathrm{NMR}$ spectra of authentic and adulterated Curcuma longa with Curcuma heyneana in various adulterant concentrations in the aromatic region

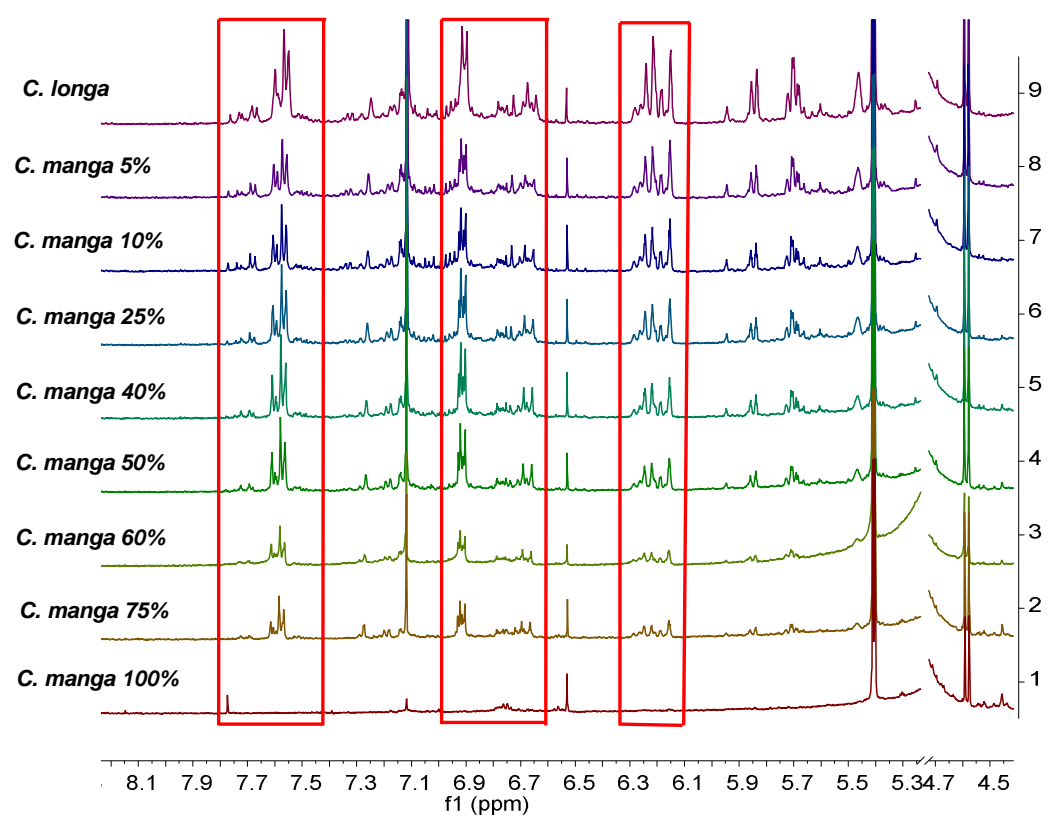

Fig. 3: ${ }^{1} \mathrm{H}-\mathrm{NMR}$ spectra of authentic and adulterated Curcuma longa with Curcuma manga in various adulterant concentrations in aromatic region

${ }^{1} \mathrm{H}-\mathrm{NMR}$ spectroscopy and chemometrics supervised pattern recognition of PLS-DA and OPLS-DA for authentication of Curcuma longa

Original ${ }^{1} \mathrm{H}-\mathrm{NMR}$ spectra contain thousands of variables. Because of the complexity of the data, it is very difficult to analyze the spectra visually. Chemometrics such as supervised pattern recognition offers some advantages to manage huge data generated from NMR spectroscopy. PLS-DA was performed to distinguish between authentic $C$. longa and adulterated $C$. longa with $C$. heyneana and $C$. manga. PLS-DA is a supervised pattern recognition which allows to search latent variables in a dataset to obtain maximum variation [25]. PLS-DA was carried out using 8 principal components explaining the total variance of $97.9 \%$. PLS-DA score plot using PC1 and PC2 showed a good differentiation between authentic $C$. longa and adulterated $C$. longa with $C$. heyneana and $C$. manga as shown in fig. 4. The measured R2X, R2Y, and Q2 were 0.979, 0.98, and 0.706, respectively. The high value of $\mathrm{R} 2 \mathrm{X}$ and $\mathrm{R} 2 \mathrm{Y}$ (close to 1) indicated the goodness of fit of the model and the Q2 value (higher than 0.5 ) indicated the goodness of predictivity of the model [21]. 


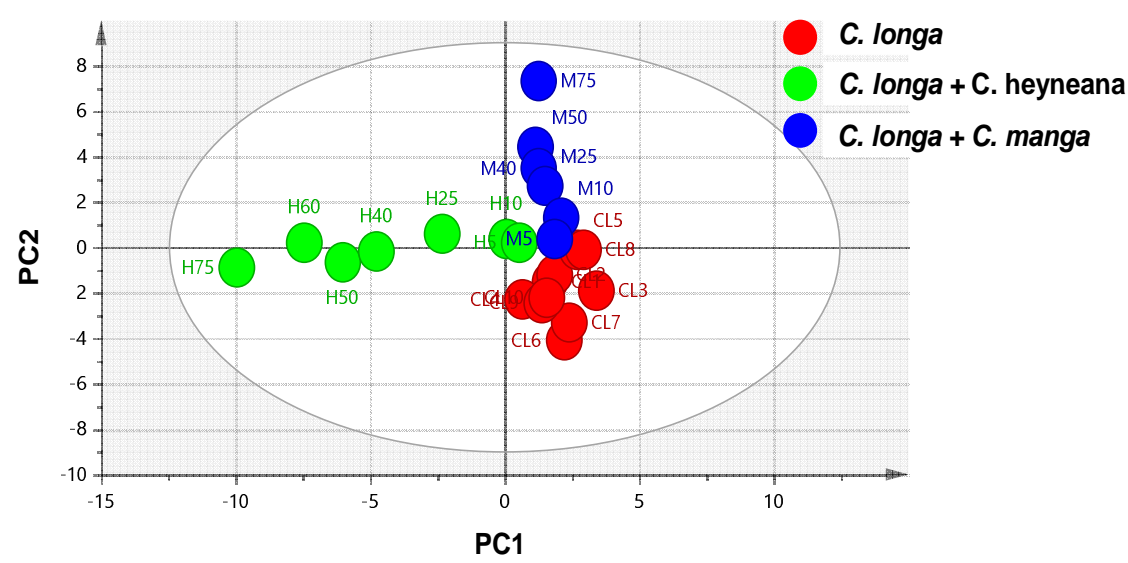

Fig. 4: PLS-DA score plot of Curcuma longa and adulterated Curcuma longa with Curcuma heyneana and Curcuma manga

Supervised method of OPLS-DA was successfully applicated to differentiate between authentic and adulterated $C$. longa with $C$. heyneana and $C$. manga, and it provided better separation than PLSDA as shown in fig. 5. OPLS-DA was carried out using two predictive and five orthogonal components. The high value of R2X (0.867) and R2Y (0.92) showed the goodness of fit of the model and the Q2 (0.521) showed the goodness of predictivity of the model. OPLS-DA showed a better performance for differentiation than PLS-DA because OPLS-DA incorporates orthogonal signal correction (OSC) filter that allows separation of $\mathrm{Y}$ predictive and $\mathrm{Y}$ orthogonal. Variables not correlated to $\mathrm{Y}$ predictive are removed so that the noise is minimized and only variables correlated to $\mathrm{Y}$ are used to build the model. Therefore, maximum separation is obtained indicating the better prediction performance [26].

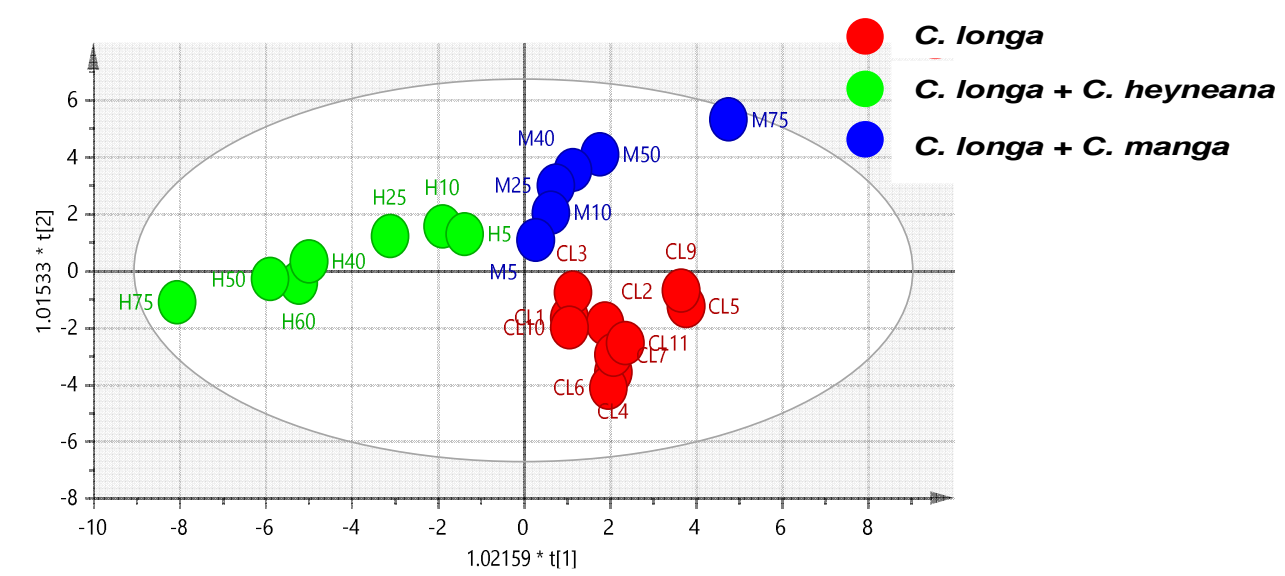

Fig. 5: OPLS-DA score plot of Curcuma longa and adulterated Curcuma longa with Curcuma heyneana and Curcuma manga

Although PLS-DA and OPLS-DA have a great performance for classification, they have a tendency to be overfitting which can cause misclassification. Overfitting could generate good separation even in a random dataset. Therefore, careful validation is needed to prove the model validity. A permutation test is one of the validation techniques which can be used to check the model validity. In the permutation test, the $\mathrm{X}$ variables are left intact, while the $\mathrm{Y}$ variables are permuted then the original variables were compared to the permuted variables. Original model must be higher than all the permuted models to obtain valid models. Moreover, the intersection of Q2 value must at zero or below zero. Results of permutation test confirmed the validity of the models both in PLS-DA and OPLS-DA models as shown in fig. 6a and b, respectively. The original models of Q2 and R2 values were higher than all permuted models both in PLS-DA and OPLS-DA models, and the intersection of Q2 was $(0,-0.991)$ in PLS-DA and $(0,-0.79)$ in OPLSDA indicated the good quality of the models.

${ }^{1}$ H-NMR-based metabolite fingerprinting and partial least square (PLS) regression for quantification of adulterants in Curcuma longa

Partial least square (PLS) is one of the regression methods in multivariate analysis with some desirable advantages. PLS is based on the features of principal component analysis and multiple regression. PLS can be used to quantify the concentration of adulteration from large data. PLS allows to analyze data with more variables than observations, and it has a lower risk in overfitting [27]. Therefore, it has been widely used in food and plants medicinal science for authentication. Quantification of adulterants of $C$. heyneana and C. manga in binary mixtures with C. longa was performed using PLS model in the concentration range of 0-100\% wt/wt. Fig. 7 showed the PLS response plot which correlates the actual and calculated response of $C$. heyneana in $C$. longa obtained from ${ }^{1} \mathrm{H}$-NMR spectra. The actual response corresponds to the actual concentration of $C$. heyneana in C. longa while the calculated response is the concentration of $C$. heyneana predicted by the model. The $\mathrm{R}^{2}$ value showed the proportion of variation in each response that is explained by the predictors. Result showed that a good relationship was obtained between actual and calculated response with the equation of $y=0.9992 x+0.0344$ and the $R^{2}$ value was 0.9992, indicating that the model fits the data well. PLS response plot of actual response and calculated response of $C$. manga in binary mixtures with $C$. longa was shown in fig. 8 . There was a linear relationship between the actual and calculated response $\left(R^{2}=0.9982\right)$ with the equation of $y=0.9982 x+0.0745$. 
a

R2

$\square \mathrm{Q} 2$

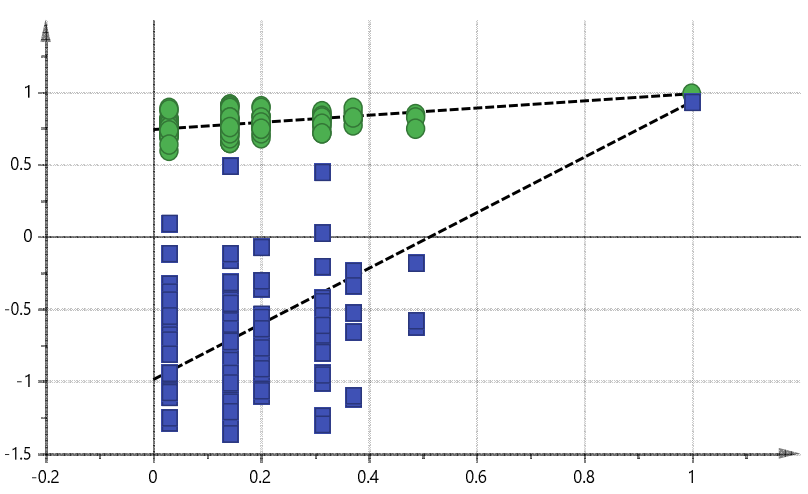

b

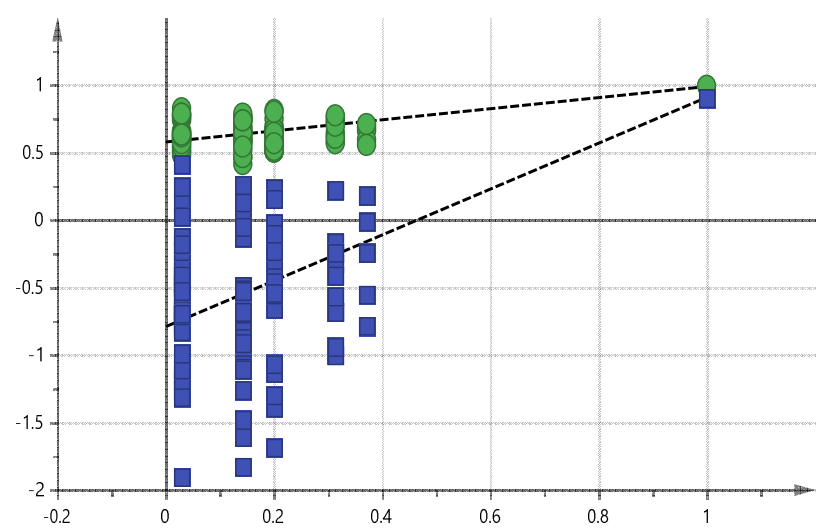

Fig. 6: Permutation test of PLS-DA (a) and OPLS-DA (b) models

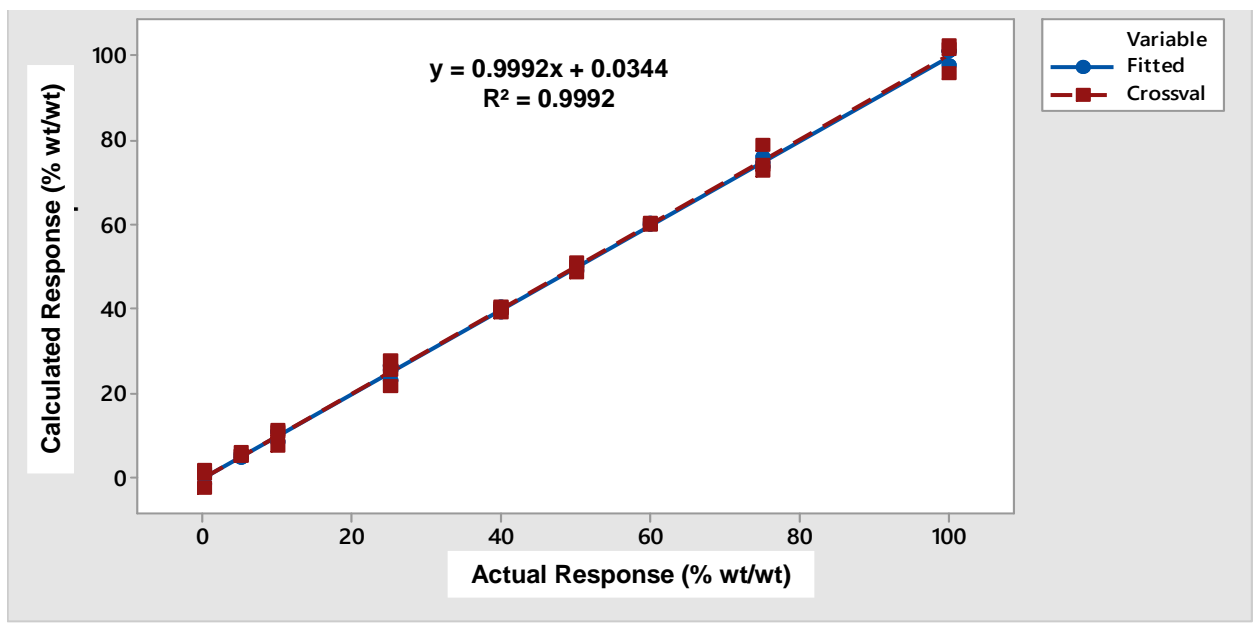

Fig. 7: PLS response plot of actual and predicted concentration of Curcuma heyneana in binary mixtures with Curcuma longa

The PLS models were evaluated using the root mean square error of calibration (RMSEC) value as the validity criteria for the calibration model. The obtained RMSEC values were $0.94 \%$ and $1.37 \%$ for the calibration model of $C$. heyneana in $C$. longa and C. manga in C. longa, respectively. RMSEC is a parameter to evaluate the occurrence of overfitting and to make a prior assumption that the relationship is in a linear way. The lower RMSEC values indicated the high accuracy and precision of the models [27]. The PLS models were validated using leave one out cross-validation technique. The predicted $\mathrm{R}^{2}$ value was used to evaluate cross-validation. It tells how well each calculated model predicts the response. Cross-validation selects the number of components which provide the most accurate predictive model. In the
PLS model of adulterated $C$. longa with $C$. heyneana, cross-validation selected ten components and it produced the highest predicted $\mathrm{R}^{2}$ value $(0.9968)$ while in the PLS model of adulterated $C$. longa with $C$. manga, cross-validation selected five components with the predicted $\mathrm{R}^{2}$ value of 0.9892 . The models were also validated using the validation model. The quality of the validation model was evaluated using RMSEP (root mean square error of prediction) value. It can give the proof of good predictive performance. The RMSEP values for validation model of $C$. heyneana in $C$. longa and $C$. manga in $C$. longa were $0.83 \%$ and $1.34 \%$, respectively. The small difference between RMSEC and RMSEP indicated the goodness of fit of the model and model overfitting did not occur. 


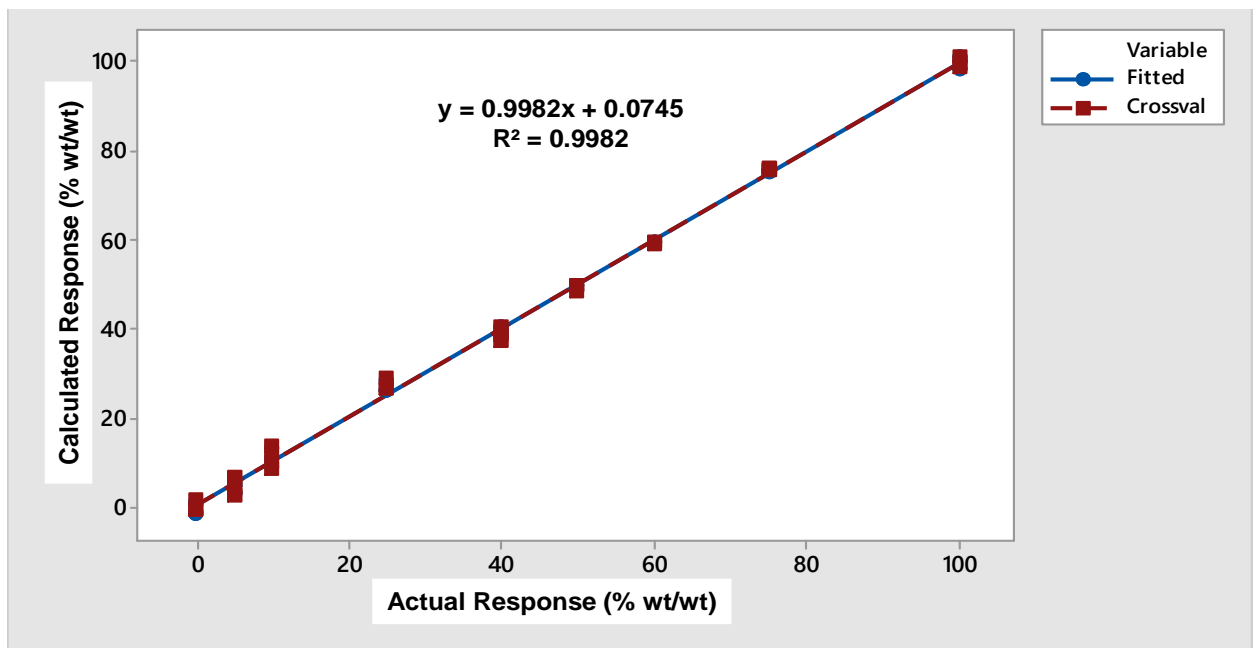

Fig. 8: PLS response plot of actual and predicted concentration of Curcuma manga in binary mixtures with Curcuma longa

\section{CONCLUSION}

Chemometrics supervised pattern recognition of PLS-DA and OPLS-DA proves to be very useful for differentiation of $C$. longa and adulterated $C$. longa with C. heyneana and C. manga. Chemometrics regression of PLS showed an adequate technique for the detection and quantification of adulteration in C. longa with $C$. heyneana and C. manga in binary mixtures. These results strongly support that combination of ${ }^{1} \mathrm{H}-\mathrm{NMR}$ based metabolite fingerprinting and chemometrics of PLS-DA, OPLS-DA and PLS is a powerful method for the authentication of $C$. longa.

\section{ACKNOWLEDGMENT}

The author thanks to Faculty of Pharmacy Universitas Gadjah Mada for funding this research, Indonesian Endowment Fund for Education Scholarship (LPDP) for supporting, and Integrated Research and Testing Laboratory (LPPT) Universitas Gadjah Mada for facilitating instrument in this research.

\section{AUTHORS CONTRIBUTIONS}

All the author have contributed equally

\section{CONFLICT OF INTERESTS}

The author declares that there is no conflict of interest

\section{REFERENCES}

1. Pothitirat W, Gritsanapan W. Quantitative analysis of curcumin, demethoxycurcumin, and bisdemethoxycurcumin in the crude curcuminoid extract from Curcuma longa in Thailand by TLCdensitometry. Mahidol Univ J Pharm Sci 2005;32:23-30.

2. Dall'Acqua S, Stocchero M, Boschiero I, Schiavon M, Golob S, Uddin J, et al. New findings on the in vivo antioxidant activity of Curcuma longa extract by an integrated 1H-NMR and HPLC-MS metabolomic approach. Fitoterapia 2016;109:125-31.

3. Cousins M, Adelberg J, Chen F, Rieck J. Antioxidant capacity of fresh and dried rhizomes from four clones of turmeric Curcuma longa L. grown in vitro. Ind Crops Prod 2007;25:129-35.

4. Mohanty I, Singh Arya D, Dinda A, Joshi S, Talwar KK, Gupta SK. Protective effects of Curcuma longa on ischemia-reperfusioninduced myocardial injuries and their mechanisms. Life Sci 2004;75:1701-11.

5. Li S, Yuan W, Deng G, Wang P, Yang P, Aggarwal B. Chemical composition and product quality control of turmeric Curcuma longa L. Pharm Crops 2011;2:28-54.

6. Bar-Sela G, Epelbaum R, Schaffer M. Curcumin as an anti-cancer agent: a review of the gap between basic and clinical applications. Curr Med Chem 2010;17:190-7.

7. Shi T, Zhu M, Chen Y, Yan X, Chen Q, Wu X, et al. $1 \mathrm{H}$ NMR combined with chemometrics for the rapid detection of adulteration in camellia oils. Food Chem 2018;242:308-15.
8. Remya R, Syamkumar S, Sasikumar B. Isolation and amplification of DNA from turmeric powder. $\mathrm{Br}$ Food J 2004;106:673-8.

9. Singh RSP, Das U, Dimmock JR, Alcorn J. A general HPLC-UV method for the quantitative determination of curcumin analogues containing the 1,5-diaryl-3-oxo-1,4-pentadienyl pharmacophore in rat biomatrices. J Chromatogr B 2010;878:2796-802.

10. Long $\mathrm{Y}$, Zhang $\mathrm{W}$, Wang F, Chen Z. Simultaneous determination of three curcuminoids in Curcuma longa L. by highperformance liquid chromatography coupled with electrochemical detection. J Pharm Anal 2014;4:325-30.

11. Phattanawasin P, Sotanaphun U, Sriphong L. Validated TLC image analysis method for simultaneous quantification of curcuminoids in Curcuma longa. Chromatographia 2009;69:397-400.

12. Ashraf K, Mujeeb M, Ahmad A, Amir M, Mallick MN, Sharma D. Validated HPTLC analysis method for quantification of variability in the content of curcumin in Curcuma longa L (turmeric) collected from the different geographical region of India. Asian Pac J Trop Biomed 2012;2:S584-8.

13. Cheng J, Weijun K, Yun L, Jiabo W, Haitao W, Qingmiao L, et al. Development and validation of UPLC method for quality control of Curcuma longa Linn.: fast simultaneous quantitation of three curcuminoids. J Pharm Biomed Anal 2010;53:43-9.

14. Anubala S, Sekar R, Nagaiah K. Development and validation of an analytical method for the separation and determination of major bioactive curcuminoids in Curcuma longa rhizomes and herbal products using non-aqueous capillary electrophoresis. Talanta 2014;123:10-7.

15. Sharma S, Saraogi GK, Kumar V. Development of spectrophotometric methods for simultaneous determination of artesunate and curcumin in a liposomal formulation. Int J App Pharm 2015;7:18-21.

16. Al-Taani B, Khanfar M, Alsuod OA. Enhancement of the release of curcumin by the freeze-drying technique using inulin and neusilin as carriers. Int J Appl Pharm 2018;10:42-8.

17. Wang L, Lee F, Wang X, He Y. Feasibility study of quantifying and discriminating soybean oil adulteration in camellia oils by attenuated total reflectance MIR and fiber optic diffuse reflectance NIR. Food Chem 2006;95:529-36.

18. Verpoorte R, Choi YH, Kim HK. NMR-based metabolomics at work in phytochemistry. Phytochem Rev 2007;6:3-14.

19. Kim HK, Saifullah N, Khan S, Wilson EG, Kricun SDP, Meissner A, et al. Metabolic classification of South American Ilex species by NMR-based metabolomics. Phytochemistry 2010;71:773-84.

20. Petrakis EA, Cagliani LR, Polissiou MG, Consonni R. Evaluation of saffron Crocus sativus L. adulteration with plant adulterants by 1H-NMR metabolite fingerprinting. Food Chem 2015; 173:890-6. 
21. Gogna N, Hamid N, Dorai K. Metabolomic profiling of the phytomedicinal constituents of Carica papaya L. leaves and seeds by $1 \mathrm{H}-\mathrm{NMR}$ spectroscopy and multivariate statistical analysis. J Pharm Biomed Anal 2015;115:74-85.

22. Fadzillah NA, Che Man YB, Rohman A, Rosman AS, Ismail A, Mustafa $S$, khatib A. Detection of butter adulteration with lard by employing ${ }^{1} \mathrm{H}-\mathrm{NMR}$ spectroscopy and multivariate data analysis. J Oleo Sci 2015;64:697-703.

23. Awin T, Mediani A, Maulidiani, Shaari K, Faudzi SMM, Sukari $\mathrm{MAH}$, et al. Phytochemical profiles and biological activities of Curcuma species subjected to different drying methods and solvent systems: NMR-based metabolomics approach. Ind Crops Prod 2016;94:342-52.
24. Bos R, Windono T, Woerdenbag HJ, Boersma YL, Koulman A, Kayser O. HPLC-photodiode array detection analysis of curcuminoids in Curcuma species indigenous to Indonesia. Phytochem Anal 2007;18:118-22.

25. Worley B, Halouska S, Powers R. Utilities for quantifying separation in PCA/PLS-DA scores plots. Anal Biochem 2013;433:102-4.

26. Worley B, Powers R. Multivariate analysis in metabolomics. Curr Metabolomics 2013;1:92-107.

27. Rohman A, Che Man YB. The optimization of FTIR spectroscopy combined with partial least square for analysis of animal fats in quartenary mixtures. J Spectrosc 2011;25:169-76. 